\title{
Topography of selectively laser melted surfaces: A comparison of different measurement methods
}

\author{
Adam Thompson a, Nicola Senin a,b, Claudiu Giusca c, Richard Leach (2)a \\ a Manufacturing Metrology Team, University of Nottingham, NG7 2RD, UK \\ ${ }^{b}$ Department of Engineering, University of Perugia, 06125, Italy \\ c Precision Engineering Institute, Cranfield University, MK43 OAL, UK
}

Selective laser melting (SLM) of metals produces surface topographies that are challenging to measure. Multiple areal surface topography measurement technologies are available, which allow reconstruction of information rich, three-dimensional digital surface models. However, the capability of such technologies to capture intricate topographic details of SLM parts has not yet been investigated. This work explores the topography of a SLM Ti6Al4V part, as reconstructed from measurements by various optical and non-optical technologies. Discrepancies in the reconstruction of local topographic features are investigated through alignment and quantitative assessment of local differences. ISO 25178-2 areal texture parameters are computed as further comparison indicators.

Metrology, Surface analysis, Selective Laser Melting (SLM)

\section{Introduction}

Recent developments in areal topography measurement have enabled the fast acquisition of high-density surface datasets, allowing the reconstruction of detailed three-dimensional digital models of surface topography. There are a number of industrially recognised optical technologies for areal topography measurement; confocal microscopy (CM) [1], coherence scanning interferometry (CSI) [2] and focus variation (FV) microscopy [3] being the most prominent. Optical areal measurement technologies are becoming increasingly accepted alongside conventional profile measurement via contact stylus [4]. Recent work has also shown X-ray computed tomography (XCT) to be capable of capturing topographical information at scales approaching those captured by the optical methods $[5,6]$.

Surface topographies produced by selective laser melting (SLM) of metals are highly complex [7-11], featuring relevant topographic detail at multiple scales, with a mixture of high and low aspect-ratio formations, high slopes, undercuts and deep recesses, in particular for lower-density builds. Surfaces also often feature non-uniform optical properties due to local oxidisation effects and the presence of complex micro-scale topographic patterns. SLM surfaces are typically highly challenging for any type of areal measurement technology, optical or non-optical [12].

Preliminary work performed by the authors on the measurement of AM surfaces indicated that, for SLM surfaces, areal topography data obtained with different technologies show significant discrepancies. Thus far, however, the investigation has been conducted from a mostly qualitative standpoint [13]. Differences between reconstructed topographic details sometimes appear to be of the same order of magnitude as the topographic features being investigated. In some instances, the overall shape of a topographic feature is reconstructed in an entirely different manner depending on the measurement technology: small recesses may become protrusions, while regular hemispheric shapes (e.g. spatter particles) may appear as completely irregular [13]. The problem of assessing the metrological performance of the available optical, areal measurement technologies is as of yet unsolved. Little is currently known about how to quantify and correct bias in topographic reconstruction, or how to evaluate the associated uncertainty [14]. Research regarding the use of XCT for topographical measurement is even more in its infancy. Even for dimensional characterisation tasks, XCT measurement is generally hampered by numerous challenges [15].

A significant part of the problem of assessing the measurement error associated with the different technologies is the lack of a traceable reference measurement. For complex topography, the only technology that can be relatively easily employed as a reference is profile measurement via contact stylus [12]. However, the problem of how to reliably relocate profile data measured by a stylus onto areal topography data remains unsolved. Even in the case of successful relocation, the relocated profile only allows comparison with the cross-section of the areal topography dataset, and it is often not convenient to cover a large measured area with multiple stylus profiles due to the large amount of time required to scan multiple profiles at sufficient spatial resolution.

In the absence of a reliable reference to assess measurement error in areal topography data, it is still possible to compare surface measurements in terms of agreement and disagreement. This approach has been recently attempted for injection moulded replicates of nickel transfer standards, although comparison focused solely on the observation of texture parameters [16]. Texture parameters are important, and represent the only method currently used by the design and manufacturing communities to describe topographies. Efforts are, therefore, justified in providing measures of agreement and disagreement in terms of texture parameters. However, only comparative analysis of reconstructed topographies allows for acquisition of an understanding as to why calculated parameters differ.

In this work, the surface of an SLM sample is acquired with multiple topography measurement instruments. Topographies are 
relocated for the purpose of direct, quantitative comparison of reconstructed local features. Areal texture parameters are also computed and discrepancies discussed, starting from the differences observed in the reconstructed topographies.

\section{Methodology}

\subsection{Sample}

A $(20 \times 20 \times 20) \mathrm{mm}$ Ti6Al4V cubic artefact, manufactured using SLM with a Renishaw AM 250, was used as the test case. A portion of the top surface (orthogonal to the building direction) was considered, located near the cube edges to facilitate relocation. The surface was not post-processed so as to preserve the typical topographic features generated by SLM.

\subsection{Measurement set-ups}

Four commercial measurement instruments were used, each representative of a relevant measurement technology (CM, FV, CSI and XCT). Instrument names have been redacted to prevent direct comparison of commercial instruments. The sample was measured three times consecutively with each instrument, with no refixturing between measurements, constant set-up parameters, and controlled temperature environments $\left(\mathrm{CM}: \pm 0.1^{\circ} \mathrm{C}, \mathrm{CSI}\right.$ and FV: $\pm 1{ }^{\circ} \mathrm{C}, \mathrm{XCT}: \pm 0.2^{\circ} \mathrm{C}$ ). The following set-ups were adopted:

- CM: 20x objective lens (NA 0.6, field of view-FoV $(0.64 \times 0.64) \mathrm{mm})$; lateral resolution (pixel width) $0.625 \mu \mathrm{m}$; lateral resolution (optical limit): $0.12 \mu \mathrm{m}$; measured area: (2.9x 2.9) $\mathrm{mm}$, stitched;

- CSI: $20 \times$ objective at $1 \times$ zoom $(\mathrm{NA} 0.4$, FoV $(0.42 \times 0.42) \mathrm{mm})$ lateral resolution (pixel width) $0.409 \mu \mathrm{m}$; lateral resolution (optical limit): $0.71 \mu \mathrm{m}$; measured area: $(3.4 \times 3.4) \mathrm{mm}$, stitched;

- FV: $20 \times$ objective lens $(\mathrm{NA} 0.4$, FoV $(0.81 \times 0.81) \mathrm{mm})$ lateral resolution (pixel width) $0.439 \mu \mathrm{m}$; lateral resolution (optical limit): $0.88 \mu \mathrm{m}$; ring light illumination [17]; measured area: (3.7 × 3.7), stitched;

- XCT: geometric magnification of $42.6 \times$ leading to a voxel size of $4.69 \mu \mathrm{m}, 3142 \mathrm{X}$-ray projections (formed from averaging of two exposures per projection, each lasting $2 \mathrm{~s}$ ); tube voltage $150 \mathrm{kV}$; current $30 \mu \mathrm{A} ; 1 \mathrm{~mm}$ copper pre-filter. Data were reconstructed in the manufacturer's proprietary software, using no beam hardening correction. Surfaces (triangulated meshes) were determined in VGStudio MAX 3.0 [18] from volumetric data, using the maximum gradient method [19].

The chosen set-ups for each instrument represent the results of best-practice acquired by the authors in the measurement of SLM surfaces [13]. Results discussed below may vary as a function of different measurement set-ups (magnification, illumination, etc.).

\subsection{Data processing}

\subsubsection{Data preparation}

CM, CSI and FV measurements generated height maps useable in their native forms. Triangulated meshes extracted from XCT volumetric data were converted into height maps. For the conversion, each original mesh was exported from VGStudio MAX in STL format, imported into Meshlab [20] and oriented by aligning the surface normal (obtained via principal components analysis [21]) to the $z$-axis. The result was exported as an STL and imported into MountainsMap [22] where it was raster-scanned into a height map. The spatial resolution for scanning is automatically determined by MountainsMap, to match the point density of the original triangulated mesh.

\subsubsection{Relocation and extraction}

Topography datasets were aligned in MountainsMap through rigid transformations (rotation and translation), by application of marker-based, algorithmic alignment (coarse), followed by cross correlation-based, global algorithmic alignment [23] (fine, to compensate for small misalignments caused by marker misplacement in coarse alignment). At this stage, a scaling error was recognised in the CM data and a scaling correction factor was applied, obtained by maximising alignment to other datasets. A region $(2.5 \times 2.5) \mathrm{mm}$ wide was extracted from each of the aligned datasets. The size of the region was chosen to ensure topographic significance of the sample, consistent with previous work [8].

\subsubsection{Detailed comparison of topographies}

Cross-sectional profiles were extracted from the aligned regions. For any given cross-section, this amounts to three profile replicates per instrument, for a total of twelve profiles. For each cross-section, profiles were resampled along the horizontal axis $(x)$ by linear interpolation, at the frequency corresponding to the highest lateral resolution in the original datasets so as to allow comparison whilst retaining as much of the measured information as possible. At each $x$ position, the three values of each triplicate were used to generate a confidence interval (CI) of the local mean at $95 \%$ confidence, using a $t$-distribution with two degrees of freedom (DoF), assuming normality and an absence of spatial correlation. The CIs were computed for all $x$ coordinates to obtain a mean profile and CI bands for each instrument. Discrepancy between instruments was computed as a percentage of the profile length where CIs do not overlap. Stitching of multiple datasets in optical measurement was used to obtain suitable coverage width, whilst ensuring high lateral resolution. However, it should be noted that stitching introduces uncharacterised effects in CI evaluation, due to stage repeatability and algorithmic stitching errors.

\subsubsection{Texture parameter computation}

The following areal texture parameters were considered from ISO 25178-2: Sa, Sq, Ssk, Sku and Sal [24]. Parameters were computed on surfaces where only an F-operator was applied (the F-set), and on scale-limited surfaces (the SL-set). The F-operator consisted of subtraction of a least-squares mean plane. To achieve bandwidth-matching [25] in the SL-set, an S-filter with a $6.29 \mu \mathrm{m}$ cut-off (based on a grid of $4 \times 4$ pixels in the lowest resolution dataset) and an L-filter with a $0.8 \mathrm{~mm}$ cut-off (a common filter used to separate roughness and waviness [26]) were adopted.

Parameter values computed for each dataset were aggregated by instrument type, and used to construct CIs of the mean at $95 \%$ confidence, based on $t$-distributions. Agreements and discrepancies for parameter pairs were computed by comparing confidence intervals.

\section{Results}

\subsection{Analysis of reconstructed topographies}

Figure 1 provides a qualitative overview of how the different instruments render the same topography. Figure 2 a shows twelve profiles obtained by cutting the aligned topographies in the same cross-sectioning plane. Figure $2 b$ shows a portion of the same cross-section where means and CIs are calculated for each set of profiles. Table 1 shows the discrepancy between instrument pairs, (as a percentage of the profile length where CIs do not intersect) computed on the cross-section displayed in Figure $2 \mathrm{a}$.

Table 1 Profile discrepancy between instrument pairs.

\begin{tabular}{lcccccc}
\hline & CM/CSI & CM/FV & CM/XCT & CSI/FV & CSI/XCT & FV/XCT \\
\hline Discrepancy \% & 51.7 & 61.9 & 48.5 & 57.4 & 42.9 & 48.8 \\
\hline
\end{tabular}




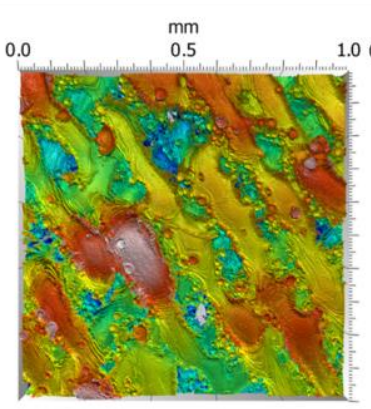

a)

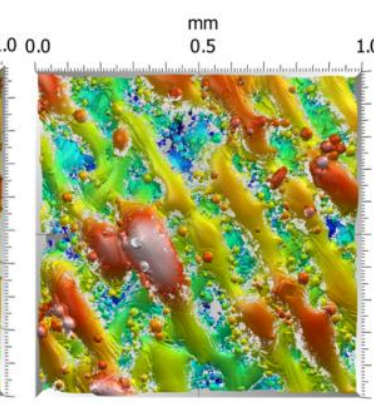

b)

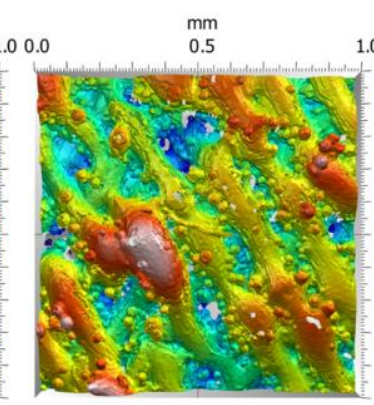

c)

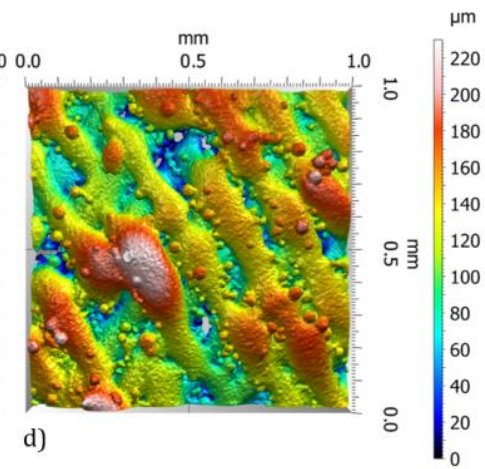

Figure 1. Reconstructed portions of aligned topographies (top views, height-based colouring); (a) CM; (b) CSI; (c) FV; (d) XCT.

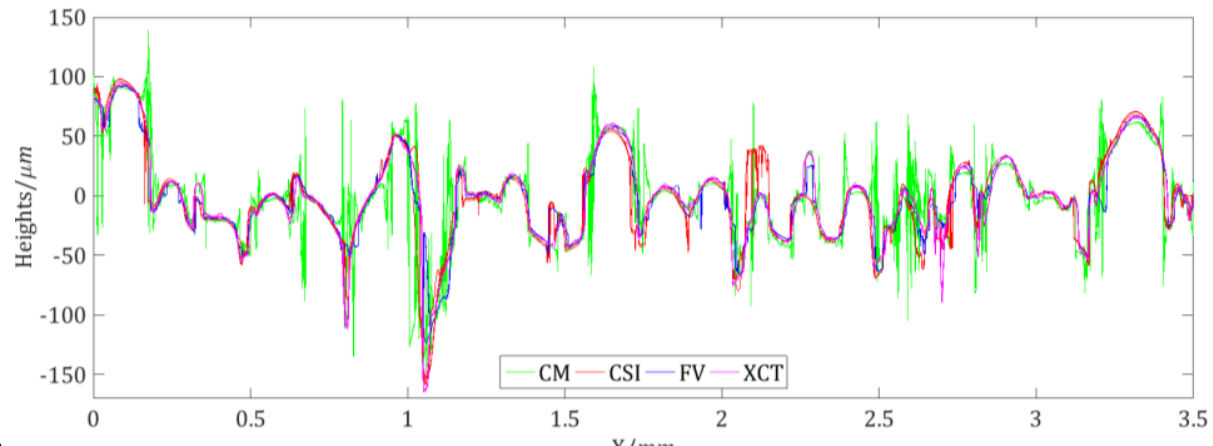

a)

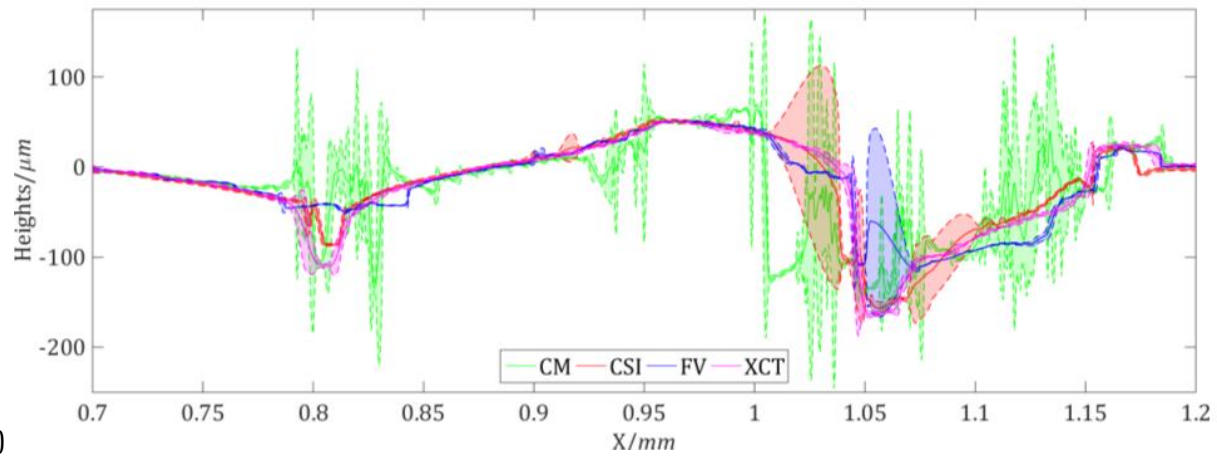

Figure 2. Profile comparison; (a) twelve profiles (three for each instrument) obtained from cross-sectioning along the diagonal of the $(2.5 \times 2.5)$ mm aligned topographies; (b) portion of the same cross-section: mean profiles and estimated CIs.

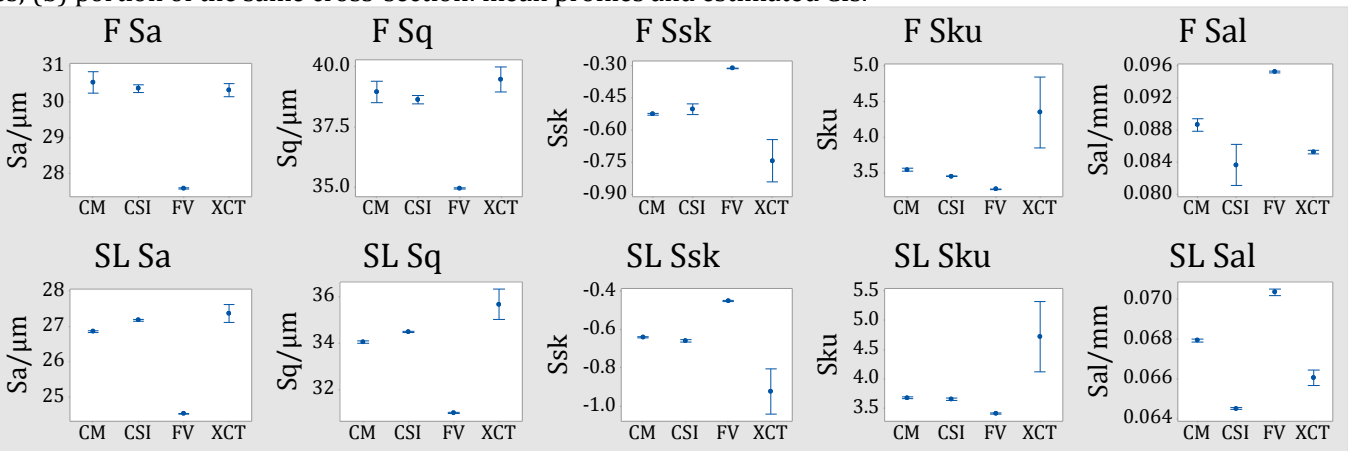

Figure 3. CIs for the texture parameters computed on the F and SL sets.

\subsection{Comparison of texture parameters}

Figure 3 shows the CI plots for the texture parameters computed on the F-set, and SL-set respectively.

\section{Discussion}

The overall discrepancy of local topographic reconstruction (Table 1) is obviously higher for those instruments with narrower CIs, i.e. better repeatability (Figure 2b). Most local topographic differences are located in difficult to measure regions such as recesses and high slopes. While some spikes can be recognised through outlier detection, this is not possible for erroneously reconstructed, wider regions (e.g. a hill instead of a recess - see CSI and FV in Figure $2 \mathrm{~b}$ ). Deep recesses are also more challenging for optical technologies, particularly $\mathrm{FV}$, which requires sufficient reflected light to compute contrast. Most areas where topographic reconstructions are in disagreement are marked by a significant increase of the CIs, suggesting that CIs from replicates may become useful indicators of local measurement reliability.

When examining texture parameters (Figure 3), the same trends are observed in the F and SL sets, meaning that the removal of very high spatial frequencies (S-filter) does not significantly affect the 
performance differences between measurement technologies (although shifts in values are observed between the F and SL sets). Overall, XCT parameter results are most frequently the least repeatable (larger CIs), although FV data sometimes exhibit similarly poor repeatability. The scarce ability of FV to capture deep recesses is confirmed by the $S s k$ and $S k u$ parameters combined, which also confirm the increased suitability of XCT for measurement of these features as observed on cross-section profiles. The most conventional type of "roughness" assessment, i.e. via the $S a$ and $S q$ parameters (analogous to the $R a$ and $R q$ ISO 4287 profile parameters), shows similar results for CM and CSI, with the XCT means coarsely located in the same region. Again, FV shows the largest discrepancy, potentially because of poorer capture of smaller scale peaks and pits. The Sal parameter confirms differences in the spatial frequencies captured by each instrument, even within the bandwidth-matched interval: FV generates the highest Sal parameter confirming the dominance of larger wavelength topographic components. CSI and XCT are the technologies that appear the most capable of acquiring higherfrequency topographic content, although Figure 1 seems to indicate that in the case of XCT, such content may actually be noise.

The results discussed until this point illustrate a few interesting points. Firstly, though texture parameters can be used to quantify differences between areal topography measurement instruments, when it comes to interpreting such results, the investigation of the aligned, reconstructed topographies is essential in order to provide information as to why specific results are obtained. The problem of accurate alignment and comparison of topographies becomes essential, with many currently unsolved challenges. Alignment is difficult in the presence of measurement error which can be as large as the topographic features of interest. Alignment results may be improved by replacing global alignment algorithms (such as cross-correlation) with selective alignment solutions based on maximising the overlap of those regions that have remained the most invariant across measurements. In turn, alignment error heavily affects statistical modelling, e.g. by unnaturally increasing the width of the CIs. Even with ideal alignment, a more statistically sound process for generating mean profiles and CIs would include spatial correlation effects, and some type of correction for simultaneous estimation of multiple CIs (e.g. Bonferroni). Both the alignment and statistical modelling problems are made more difficult by the need to handle areal data (i.e. $z=f(x, y)$ formulations), as opposed to profiles $(z=f(x))$.

\section{Conclusions and outlook}

A quantitative comparison of areal topography measurement by CM, CSI, FV and XCT technologies was performed through analysis of agreement/discrepancy of aligned topographies (profiles) and the computation of areal texture parameters. Because of the limited number of samples and set-ups investigated, the comparison should not be taken as an aid to select the most appropriate technology to measure SLM surfaces in general. However, the illustrated results bring new insight into the behaviour of measurement technologies in inspection of SLM surfaces, supporting a better-informed approach to topography inspection. The comparison method proposed in this work is applicable to multiple scenarios where comparative topography assessment is required, and can also be considered a fundamental milestone towards improving our understanding of topography measurement artefacts and their effects on texture parameters. As the proposed method for computing CIs provides, amongst other things, an indirect indication of measurement repeatability, it would be interesting to see how the information compares to surface topography repeatability as defined in ISO 25178 (parts 603-606). It would also be interesting to see if the two results could be integrated to improve understanding of the behaviour and performance of complex surface topography measurement.

\section{Acknowledgements}

AT, NS and RKL would like to thank Prof. B.M. Colosimo (Politecnico di Milano, Italy) for fruitful discussions on statistical modelling. AT and RKL would like to thank the EPSRC (Grants EP/M008983/1 and EP/L01534X/1), and 3TRPD Ltd. for funding this work. NS and RKL would also like to thank the EC for supporting this work through the grant FP7-PEOPLE-MC METROSURF. The authors would like to thank Digital Surf for providing the MountainsMap software.

\section{References}

[1] Artigas R (2011) Imaging Confocal Microscopy. in Leach R, (Ed.) Optical Measurement of Surface Topography, Springer-Verlag, Berlin, Germany237-286.

[2] de Groot P (2011) Coherence Scanning Interferometry. in Leach R, (Ed.) Optical Measurement of Surface Topography, Springer-Verlag, Berlin, Germany187-208.

[3] Helmli F (2011) Focus Variation Instruments. in Leach R, (Ed.) Optical Measurement of Surface Topography, Springer-Verlag, Berlin, Germany131-166.

[4] Leach R (2011) Introduction to Surface Texture Measurement. in Leach R, (Ed.) Optical Measurement of Surface Topography, Springer-Verlag, Berlin, Germany1- -11. [5] Townsend A, Pagani L, Scott P, Blunt LA (2017) Areal Surface Texture Data Extraction from X-ray Computed Tomography Reconstructions of Metal Addi-tively Manufactured Parts. Precision Engineering 48:254-264.

[6] Thompson A, Körner L, Senin N, Leach R, Lawes S, Maskery I (2017) Measure-ment of the Internal Surface Texture of Additively Manufactured Parts by X-ray Computed Tomography. Conference on Industrial Computed Tomography, Leu-ven, Belgium.

[7] Hong M-H, Min B, Kwon T-Y (2016) The Influence of Process Parameters on the Surface Roughness of a 3D-printed Co-Cr Dental Alloy Produced via Selective Laser Melting. Applied Science 6:401.

[8] Triantaphyllou A, Giusca CL, Macaulay GD, Roerig F, Hoebel M, Leach RK, Tomita B, Milne KA (2015) Surface Texture Measurement for Additive Manufacturing. Surface Topography: Metrology and Properties 3:24002.

[9] Grimm T, Wiora G, Witt G (2015) Characterization of Typical Surface Effects in Additive Manufacturing with Confocal Microscopy. Surface Topography: Me-trology and Properties 3:14001.

[10] Safdar A, He HZ, Wei L-Y, Snis A, Chavez de Paz LE (2012) Effect of Process Parameters Settings and Thickness on Surface Roughness of EBM Produced Ti- 6Al4V. Rapid Prototyping Journal 18:401-408.

[11] Townsend A, Senin N, Blunt L, Leach RK, Taylor JS (2016) Surface Texture Metrology for Metal Additive Manufacturing: A Review. Precision Engineering 46:3447.

[12] Leach R (2011) Optical Measurement of Surface Topography, Springer-Verlag, Berlin, Germany. [13] Thompson A, Senin N, Leach R (2016) Towards an Additive Surface Atlas. ASPE Conference: Dimensional Accuracy and Surface Finish in Additive Manufacturing, Raleigh, North Carolina.

[14] Haitjema H (2015) Uncertainty in Measurement of Surface Topography. Surface Topography: Metrology and Properties 3:35004.

[15] Kruth J-P, Bartscher M, Carmignato S, Schmitt R, De Chiffre L, Weckenmann A (2011) Computed Tomography for Dimensional Metrology. CIRP AnnalsManufacturing Technology 60:821-842.

[16] Tosello G, Haitjema H, Leach RK, Quagliotti D, Gasparin S, Hansen HN (2016) An International Comparison of Surface Texture Parameters Quantification on Polymer Artefacts Using Optical Instruments. CIRP Annals-Manufacturing Technology 65:529-532.

[17] Nikolaev N, Petzing J, Coupland J (2016) Focus Variation Microscope: Linear Theory and Surface Tilt Sensitivity. Applied Optics 55:3555. [18] Volume Graphics (2017) VGStudio MAX. Available: http://www.volumegraphics.com/en/products/vgstudio-max/ (Accessed 12 January 2017)

[19] Lifton JJ, Malcolm AA, McBride JW (2015) A Simulation-based Study on the Influence of Beam Hardening in X-ray Computed Tomography for Dimensional Metrology. Journal of X-Ray Science and Technology 23:65-82.

[20] Visual Computing Lab-ISTI-CNR, (2016), MeshLab. http://meshlab.sourceforge.net/(Accessed December 14, 2016)

[21] Jolliffe I (2014) Principal Component Analysis, Wiley, New York, USA.

[22] Digital Surf (2017) Mountains1 surface imaging \& metrology software. Available: http://www.digitalsurf.com/en/mntkey.html (Accessed 12th January 2017).

[23] Condeço J, Christensen L, Rosén B-G (2001) Software Relocation of 3D Surface Topography Measurements. International Journal of Machine Tools and Manu-facture 41:2095-2101.

[24] ISO 25178-2:2012 Geometrical Product Specifications (GPS)-Surface Texture: Areal-Part 2: Terms, Definitions and Surface Texture Parameters.

[25] Leach RK, Haitjema H (2010) Bandwidth Characteristics and Comparisons of Surface Texture Measuring Instruments. Measurement Science and Technology 21:32001.

[26] ISO 4287:1997 Geometrical Product Specification (GPS)-Surface Texture: Profile Method-Terms, Definitions and Surface Texture Parameters. 